\title{
Resonance of Double-Diffusive Convection in a Porous Medium Heated with a Sinusoidal Exciting Temperature
}

\author{
R. El Ayachi ${ }^{1}$, A. Raji ${ }^{1}$, M. Hasnaoui ${ }^{2}$, A. Abdelbaki ${ }^{2}$ and M. Naïmi ${ }^{1}$ \\ ${ }^{1}$ Sultan Moulay Slimane University, Faculty of Sciences and Technologies, Physics Department, Laboratory of Flows \\ and Transfers Modelling (LAMET), B.P. 523, Béni-Mellal 23000, Morocco \\ ${ }^{2}$ Cadi Ayyad University, Faculty of Sciences Semlalia, Physics Department, Laboratory of Fluid Mechanics and \\ Energetics (Laboratory affiliated to CNRST URAC-27), B.P. 2390, Marrakech, Morocco
}

Email: abderaji@fstbm.ac.ma and abderaji@yahoo.fr

(Received February 13, 2009; accepted July 4, 2009)

\begin{abstract}
Laminar double-diffusive convection in a two-dimensional porous square cavity differentially heated and salted is studied numerically. The left vertical wall of the cavity is heated with a temperature varying sinusoidally in time, while the opposite cold wall is maintained at a constant temperature. The same walls of the cavity are salted with constant and different concentrations (the concentration of the heated wall is higher than that of the cooled one). The remaining horizontal walls are considered adiabatic and impermeable. The parameters governing the problem are the amplitude of the variable temperature $(0 \leq a \leq 1)$, its period $(0.0001 \leq \tau \leq 10)$, the buoyancy forces ratio $(-5 \leq \mathrm{N} \leq$ $+5)$, the Lewis number $(0.1 \leq \mathrm{Le} \leq 10)$ and the thermal Darcy-Rayleigh number $\left(\mathrm{R}_{\mathrm{T}}=400\right)$. Effects of these parameters on fluid flow, temperature and concentration distributions and mean heat and mass transfers within the cavity are analyzed. Results obtained show that both heat and mass transfers could be significantly enhanced or reduced, with respect to those generated in the case of constant heating conditions by proper choice of the parameters related to the periodic temperature.
\end{abstract}

Key words: Numerical study, porous medium, double diffusive natural convection, periodic heating, resonance phenomenon

\section{NOMENClature}

a amplitude of the exciting temperature

$\mathrm{A}_{\mathrm{r}} \quad$ aspect ratio of the cavity $\mathrm{A}_{\mathrm{r}}=\mathrm{L}^{\prime} / \mathrm{H}^{\prime}$

D mass diffusivity of species, $\mathrm{m}^{2} / \mathrm{s}$

g acceleration due to the gravity, $\mathrm{m} / \mathrm{s}^{2}$

$\mathrm{K}$ permeability, $\mathrm{m}^{2}$

$\mathrm{H}^{\prime} \quad$ height of the cavity, $\mathrm{m}$

$\mathrm{L}^{\prime} \quad$ length of the cavity, $\mathrm{m}$

Le Lewis number, Eq. (7)

$\mathrm{N} \quad$ ratio of buoyancy forces, Eq. (7)

$\mathrm{Nu}(\mathrm{t}) \quad$ temporal average Nusselt number, Eq. (8)

$\overline{\mathrm{Nu}}$ time averaged Nusselt number, Eq. (9)

$\mathrm{R}_{\mathrm{T}} \quad$ thermal Rayleigh-Darcy number, Eq. (7)

$\mathrm{S}_{0}^{\prime}$ dimensional concentration imposed at the cold wall

$\mathrm{S}_{1}^{\prime}$ dimensional concentration imposed at the heated wall

$\mathrm{S}$ dimensionless concentration, $\mathrm{S}=\left(\mathrm{S}^{\prime}-\mathrm{S}_{0}^{\prime}\right) /\left(\mathrm{S}_{1}^{\prime}-\mathrm{S}_{0}^{\prime}\right)$
Sh(t) temporal Sherwood number, Eq. (8)

$\overline{\mathrm{Sh}} \quad$ time averaged Sherwood number, Eq. (9)

$\mathrm{t}$ dimensionless time, $\mathrm{t}=\mathrm{t}^{\prime} \alpha /\left(\sigma \mathrm{H}^{\prime 2}\right)$

$\mathrm{T}_{0}^{\prime} \quad$ temperature of the cold wall, $\mathrm{K}$

$\mathrm{T}_{1}^{\prime} \quad$ temperature of the heated wall, $\mathrm{K}$

$\overline{\mathrm{T}_{1}^{\prime}} \quad$ time averaged temperature of the heated wall, K

$\mathrm{T}$ dimensionless fluid temperature, $\mathrm{T}=\left(\mathrm{T}^{\prime}-\mathrm{T}_{0}^{\prime}\right) /\left(\overline{\mathrm{T}_{1}^{\prime}}-\mathrm{T}_{0}^{\prime}\right)$

$\mathrm{u}, \mathrm{v}$ dimensionless horizontal and vertical velocities, $(\mathrm{u}, \mathrm{v})=\left(\mathrm{u}^{\prime}, \mathrm{v}^{\prime}\right) \mathrm{H}^{\prime} / \alpha$

$\mathrm{x}, \mathrm{y}$ dimensionless coordinates, $(\mathrm{x}, \mathrm{y})=\left(\mathrm{x}^{\prime}, \mathrm{y}^{\prime}\right) / \mathrm{H}^{\prime}$ 


\section{Greek symbols}

$\alpha \quad$ thermal diffusivity, $\mathrm{m}^{2} / \mathrm{s}$

$\beta_{\mathrm{S}} \quad$ solutal expansion coefficient

$\beta_{\mathrm{T}} \quad$ thermal expansion coefficient, $1 / \mathrm{K}$

$\varepsilon \quad$ normalized porosity of the porous medium,

$\delta$ t $\quad \varepsilon=\varepsilon^{\prime} / \sigma$

$\lambda$ thermal conductivity of the fluid, $\mathrm{W} / \mathrm{mK}$

$v \quad$ kinematic viscosity of the fluid, $\mathrm{m}^{2} / \mathrm{s}$

$\Psi$ dimensionless stream function, $\Psi=\Psi^{\prime} / \alpha$

$\bar{\Psi}$ time averaged dimensionless stream function

$\rho \quad$ density of fluid mixture

$(\rho \mathrm{C})_{\mathrm{f}} \quad$ heat capacity of fluid

$(\rho C)_{\mathrm{P}} \quad$ heat capacity of saturated porous medium

\section{INTRODUCTION}

Combined heat and mass transfer by natural convection in porous media has been the object of intense research activity over the decades. The study of flows in porous media engendered by combined effects of thermal and mass buoyancy forces has been motivated by the importance of double diffusive convection in many natural and industrial problems. Applications implying the phenomenon cover various fields such as the migration of moisture through air contained in fibrous insulation, chemical transport in packed bed reactors, metallurgy and chemistry, grain storage, food processing and storage, contaminant transport in ground water, and many others.

A comprehensive literature review on double-diffusive natural convection in a fluid-saturated porous medium may be found in the books by Nield and Bejan (1999) and Ingham and Pop (2005). It appears that double diffusive natural convection in both vertical and horizontal enclosures completely filled with fluidsaturated porous media has been extensively studied. As far as the relation between thermal and concentration buoyancy forces is concerned, the problem of double diffusion can be classified into tree categories. For each category, heat and mass could be either aiding or opposing each other. The first category concerns the case where the porous layers are submitted to horizontal temperature and concentration gradients (see for instance (Mamou et al. 1995; Nishimura et al. 1998; Amahmid et al. 1999a; Liu et al. 2008), for the second type of configuration temperature and concentration gradients are imposed vertically (see for instance Trevisan and Bejan 1987; Amahlid et al. 1999b), while for the third configuration cross gradients of temperature and concentration (horizontal temperature gradients and vertical concentration gradients or vice versa) are imposed on the walls of the cavity (see for instance Sezai and Mohamad 1999; Bourich et al. 2004).

It is shown that heat and mass transfers are strongly depending on the governing parameters such as the ratio of the buoyancy forces, the Rayleigh-Darcy number, the Lewis number, and the aspect ratio of the cavity. In these studies, the thermal boundary conditions were assumed either steady isothermal or constant heat flux walls conditions. However, in many engineering applications, the energy provided to the $\sigma \quad$ heat capacity ratio, $\sigma=(\rho C)_{\mathrm{p}} /(\rho \mathrm{C})_{\mathrm{f}}$

$\tau \quad$ dimensionless period of the heating

temperature, $\tau=\tau^{\prime} \alpha /\left(\sigma \mathrm{H}^{\prime 2}\right)$

\section{Subscripts}

0 surface at low temperature and concentration

1 surface at high temperature and concentration

$\mathrm{p} \quad$ porous medium

f fluid

\section{Superscripts}

(') dimensional variables

(一) time averaged value

system is variable in time and gives rise to unsteady natural convection flow. In addition, heat, mass and dynamical behaviors of a porous medium subjected to time dependent thermal conditions are impossible to predict on the basis of the results obtained with constant temperature or heat flux conditions. There are many studies available in the literature concerning how a time-periodic boundary temperature affects convection in the case of fluid filled cavity (Lage and Bejan 1993; Lakhal et al. 1999; Abourida et al. 1999; Douamna et al. 2000; EL Ayachi et al. 2008). On the other hand, the studies related to the effect of temperature modulation on the onset of convection in a fluid saturated porous medium have received marginal attention (Antohe and Lage 1996; Antohe and Lage 1997; Malashetty and Wadi 1999; Malashetty and Basavaraja 2002). All these investigations were restricted to a single component fluid and porous layers.

Yet, to the best knowledge of the authors, there appears to be no studies in the literature concerned with heat and mass transfer in a porous medium heated with a periodically varying temperature. Previous studies concerned with variable periodic heating conditions were concerned with pure thermal convection. The main objective is to know how the periodicity of the imposed boundary conditions affects the timedependent flow and associated heat and mass transfers of a fluid saturated porous medium. A practical advantage is that the overall time-averaged heat and mass transfers of the system may be increased when time-varying boundary conditions are imposed. Besides this fundamental aspect of the work, this area of research is attractive and interesting since it permits to predict the system's response in numerous engineering applications involving fluid saturated porous medium such as building insulation, fire protection techniques, phase change processes, etc. Hence, the aim of this study is to investigate numerically the impact of a periodic heating temperature on thermosolutal convection developed in a square porous enclosure saturated with a binary fluid. The imposed concentrations are maintained constant. The effect of the governing parameters on fluid flow, temperature and concentration distributions and average heat and mass transfers is examined for different combinations of the governing parameters including those related to time-periodic varying temperature which are namely, its amplitude $a$ and its period $\tau$. 


\section{MAThematical Formulation}

The physical domain under investigation, sketched in Fig. 1, is a two-dimensional square enclosure saturated with a binary fluid. The square enclosure is of width $\mathrm{L}^{\prime}$ and height $\mathrm{H}^{\prime}\left(\mathrm{L}^{\prime}=\mathrm{H}^{\prime}\right)$. The Cartesian coordinates $\left(\mathrm{x}^{\prime}\right.$, $\left.\mathrm{y}^{\prime}\right)$, with the corresponding velocity components $\left(\mathrm{u}^{\prime}, \mathrm{v}^{\prime}\right)$, are indicated in Fig. 1. The problem is considered laminar and two-dimensional. The top and bottom boundaries of the enclosure are thermally insulated and impermeable. The right vertical wall of the cavity is submitted to a cold constant temperature $\mathrm{T}_{0}^{\prime}$ and to a constant concentration $\mathrm{S}_{0}^{\prime}$. The opposite left wall is submitted to a temperature $\mathrm{T}^{\prime}{ }_{1}\left(\mathrm{~T}^{\prime}{ }_{1}>\mathrm{T}^{\prime}{ }_{0}\right)$, which varies with a sinusoidal manner with time and to a constant concentration $\mathrm{S}_{1}{ }_{1}\left(\mathrm{~S}_{1}{ }_{1}>\mathrm{S}^{\prime}{ }_{0}\right)$. The Darcy model is used and the porous matrix is assumed to be homogenous and isotropic. Interaction between thermal and concentration gradients, known as the Soret and Dufour effects, are neglected. The fluid that saturates the porous matrix is modeled as a Boussinesq fluid whose density varies linearly with temperature and concentration as $\rho=\rho_{0}\left[1-\beta_{\mathrm{T}}\left(\mathrm{T}^{\prime}-\mathrm{T}^{\prime}{ }_{0}\right)-\beta_{\mathrm{S}}\left(\mathrm{S}^{\prime}-\mathrm{S}_{0}^{\prime}\right)\right]$. The dimensionless governing equations are stated as follows:

$$
\begin{aligned}
& \frac{\partial \mathrm{T}}{\partial \mathrm{t}}+\frac{\partial \mathrm{uT}}{\partial \mathrm{x}}+\frac{\partial \mathrm{vT}}{\partial \mathrm{y}}=\frac{\partial^{2} \mathrm{~T}}{\partial \mathrm{x}^{2}}+\frac{\partial^{2} \mathrm{~T}}{\partial \mathrm{y}^{2}} \\
& \varepsilon \frac{\partial \mathrm{S}}{\partial \mathrm{t}}+\frac{\partial \mathrm{uS}}{\partial \mathrm{x}}+\frac{\partial \mathrm{vS}}{\partial \mathrm{y}}=\frac{1}{\mathrm{Le}}\left(\frac{\partial^{2} \mathrm{~S}}{\partial \mathrm{x}^{2}}+\frac{\partial^{2} \mathrm{~S}}{\partial \mathrm{y}^{2}}\right) \\
& \frac{\partial^{2} \Psi}{\partial \mathrm{x}^{2}}+\frac{\partial^{2} \Psi}{\partial \mathrm{y}^{2}}=-\mathrm{R}_{\mathrm{T}}\left(\frac{\partial \mathrm{T}}{\partial \mathrm{x}}+\mathrm{N} \frac{\partial \mathrm{S}}{\partial \mathrm{x}}\right) \\
& \mathrm{u}=\frac{\partial \Psi}{\partial \mathrm{y}}, \mathrm{v}=-\frac{\partial \Psi}{\partial \mathrm{x}}
\end{aligned}
$$

Referring to Fig. 1, the dimensionless variables are:

$$
\begin{aligned}
& (\mathrm{x}, \mathrm{y})=\left(\frac{\mathrm{x}^{\prime}}{\mathrm{H}^{\prime}}, \frac{\mathrm{y}^{\prime}}{\mathrm{H}^{\prime}}\right), \mathrm{S}=\frac{\left(\mathrm{S}^{\prime}-\mathrm{S}_{0}^{\prime}\right)}{\left(\mathrm{S}_{1}^{\prime}-\mathrm{S}_{0}^{\prime}\right)}, \\
& \Psi=\frac{\Psi^{\prime}}{\alpha}, \mathrm{T}=\frac{\left(\mathrm{T}^{\prime}-\mathrm{T}_{0}^{\prime}\right)}{\left(\mathrm{T}_{1}^{\prime}-\mathrm{T}_{0}^{\prime}\right)} \text { and } \\
& (\mathrm{u}, \mathrm{v})=\left(\frac{\mathrm{u}^{\prime} \mathrm{H}^{\prime}}{\alpha}, \frac{\mathrm{v}^{\prime} \mathrm{H}^{\prime}}{\alpha}\right)
\end{aligned}
$$

The hydrodynamic boundary conditions are such that the stream function is zero on the rigid walls of the cavity $(\Psi=0)$. The dimensionless thermal and solutal boundary conditions of temperature and concentration associated to the governing equations are:

$$
\begin{array}{ll}
\mathrm{T}=1+a \sin (2 \pi \mathrm{t} / \tau), \mathrm{S}=1 \quad \text { for } \mathrm{x}=0 \\
\text { and } 0 \leq \mathrm{y} \leq 1 & \text { for } \mathrm{x}=1 \text { and } 0 \leq \mathrm{y} \leq 1 \\
\mathrm{~T}=0, \mathrm{~S}=0 & \text { on the horizontal walls } \\
\frac{\partial \mathrm{T}}{\partial \mathrm{y}}=\frac{\partial \mathrm{S}}{\partial \mathrm{y}}=0 &
\end{array}
$$

where $a$ and $\tau$ are the dimensionless amplitude and period of the heating temperature, respectively.

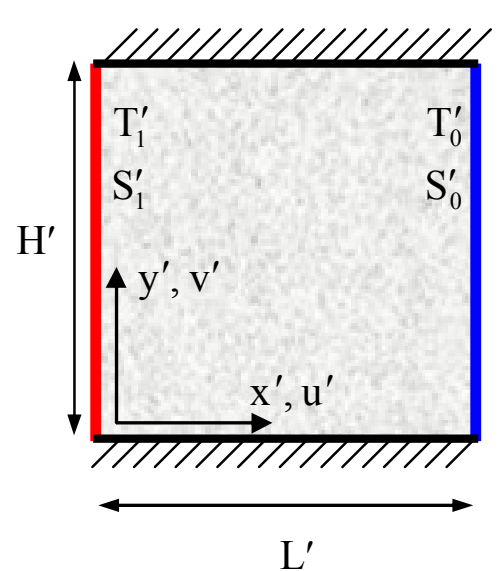

(a)

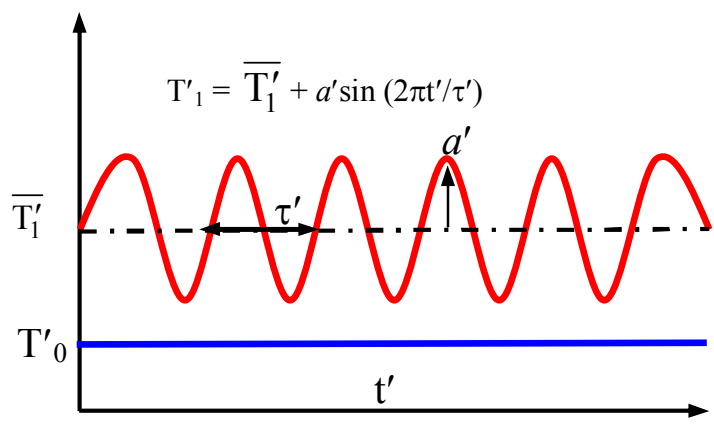

(b)

Fig. 1. (a) Geometry of the problem. (b) Thermal excitations.

In the above equations, the parameters $\mathrm{Le}, \mathrm{R}_{\mathrm{T}}$ and $\mathrm{N}$ are defined as:

$$
\begin{aligned}
& \mathrm{Le}=\frac{\alpha}{\mathrm{D}}, \mathrm{R}_{\mathrm{T}}=\frac{\mathrm{g} \cdot \beta_{\mathrm{T}} \cdot \mathrm{K} \cdot\left(\overline{\mathrm{T}_{1}^{\prime}}-\mathrm{T}_{0}^{\prime}\right) \mathrm{H}^{\prime}}{\alpha \cdot v} \text { and } \\
& \mathrm{N}=\frac{\beta_{\mathrm{S}} \cdot\left(\mathrm{S}_{1}^{\prime}-\mathrm{S}_{0}^{\prime}\right)}{\beta_{\mathrm{T}} \cdot\left(\overline{\mathrm{T}_{1}^{\prime}}-\mathrm{T}_{0}^{\prime}\right)}
\end{aligned}
$$

The thermal Rayleigh-Darcy number $R_{T}$ is defined similarly as in single natural convection heat transfer problems in porous enclosures, while the buoyancy ratio, $\mathrm{N}$, and the Lewis number, Le, arise in double diffusive natural convection problems. The buoyancy ratio $\mathrm{N}$ denotes the relative strengths of solutal and thermal buoyancy forces. It can be either positive or negative; its sign depends rather on the ratio of $\beta_{\mathrm{S}}$ and $\beta_{\mathrm{T}}$. The volumetric expansion coefficient due to temperature change is normally positive, but the volumetric expansion coefficient for concentration can be either positive $(\mathrm{N}>0)$ or negative $(\mathrm{N}<0)$. For the boundary conditions prescribed by Eq. (6), thermal and solutal buoyancy effects are aided/(opposite) when $\mathrm{N}$ is positive/(negative). Further, $\mathrm{N}$ is zero for negligible species effect (case of pure thermal convection) and infinite for solute dominating effects.

From the aforementioned boundary conditions, it appears that the present problem is governed by two additional dimensionless parameters which are the amplitude $a$ and the period $\tau$ of the exciting temperature. 


\section{Heat and Mass Transfer Evaluation}

At each time step, heat and mass transfers which are characterized by mean Nusselt and Sherwood numbers through the heated wall are evaluated as:

$$
\mathrm{Nu}(\mathrm{t})=-\left.\int_{0}^{1} \frac{\partial \mathrm{T}}{\partial \mathrm{x}}\right|_{\mathrm{x}=0} \text { dy } \operatorname{Sh}(\mathrm{t})=-\left.\int_{0}^{1} \frac{\partial \mathrm{S}}{\partial \mathrm{x}}\right|_{\mathrm{x}=0} \mathrm{dy}
$$

The mean Nusselt and Sherwood numbers, averaged in time over periods, are respectively defined as:

$$
\overline{\mathrm{Nu}}=\frac{1}{\tau_{\mathrm{Nu}}} \int_{0}^{\tau_{\mathrm{Nu}}} \mathrm{Nu}(\mathrm{t}) \mathrm{dt} \quad \overline{\mathrm{Sh}}=\frac{1}{\tau_{\mathrm{Sh}}} \int_{0}^{\tau_{\mathrm{Sh}}} \mathrm{Sh}(\mathrm{t}) \mathrm{dt}
$$

where $\tau_{\mathrm{Nu}}$ and $\tau_{\mathrm{Sh}}$ represent the periods of the temporal variations of Nusselt and Sherwood numbers (they are identical in general), respectively.

\section{Numerical Method}

The conservation Eqs. (1) to (4) were discretized by using a second order accurate finite difference method. The integration of Eqs. (1) and (2) was ensured by the Alternate Direction Implicit method (ADI). Values of the stream function at all grid points were obtained with Eq. (3) by using the Point Successive Over-Relaxation method (PSOR) with an optimum over-relaxation coefficient equal to 1.90 for the uniform grid of $81 \times 81$ adopted in the present study. Variations of $\Psi$ lower than $10^{-4}$ were adopted as a convergence criterion at each time step. A further decrease of this value did not cause any significant change in the results. The values of time step sizes considered for the simulations were selected between $2 \times 10^{-6}$ and $2 \times 10^{-3}$. Finally, the accuracy of the numerical model was checked by comparing results from the present code with those previously published by Goyeau et al. 1996, Bennacer et al. 2001, Bourich et al. 2004 and recently by Liu et al. 2008 in the case of double diffusive natural convection in square porous enclosures. The comparative results, presented in Table 1, are obtained in a square cavity differentially heated and salted and having impermeable and adiabatic horizontal walls with $\mathrm{R}_{\mathrm{T}}$ varying in the range $100 \leq \mathrm{R}_{\mathrm{T}} \leq 400$ and $\mathrm{Le}=10$. Computed heat and mass transfer rates are found to be in excellent agreement with those reported in refs. (Goyeau et al. 1996, Bennacer et al. 2001, Bourich et al. 2004 and recently by Liu et al., 2008); the maximum relative differences being less than $2.03 \%$ and $3.52 \%$ in terms of Nusselt and Sherwood numbers, respectively. Thermal and solutal boundary conditions were modified to reproduce the results recently presented by Liu et al. 2008 in the case of double-diffusive natural convection within a vertical porous enclosure locally heated and salted from one side. Good agreement can be seen from Table 2 with maximum deviation of about $3.63 \%$. Another test of validation has been done by proceeding to a systematic verification of heat and mass balances for the system. In fact, at each flow cycle, the mean quantities of heat and mass supplied by the left wall (x $=0$ ) to the system must leave the computation domain through its right surface $(x=1)$ since the horizontal walls are impermeable and adiabatic. These thermal and solutal balances have been verified within $0.2 \%$ and
$0.3 \%$, respectively. Details relative to the sensitivity of the results vis-à-vis the grid, given in Table 3, justify the selected grid of $81 \times 81$ as a reasonable compromise between computational effort and required accuracy. More precisely, the maximum deviations observed in terms of $\mathrm{Nu}$, Sh and $\Psi_{\max }$ remained within $0.18 \%$, $2.22 \%$ and $1.30 \%$, respectively, when the $81 \times 81$ grid was refined to $101 \times 101$.

\section{Results AND Discussion}

The foregoing analysis shows that the problem is governed by a high number of parameters $\left(\mathrm{A}_{\mathrm{r}}, \mathrm{R}_{\mathrm{T}}, \mathrm{N}\right.$, Le, $\varepsilon, a$ and $\tau$ ) which renders unrealistic a full blown parametric investigation. Hence, illustrative results on heat and mass transfer characteristics are presented for $\mathrm{A}_{\mathrm{r}}=1, \mathrm{R}_{\mathrm{T}}=400$, and $\varepsilon=0.8$. The choice of this value of $R_{T}$ was based on a preliminary study conducted with various values of this parameter leading to very limited qualitative changes on the obtained results. Effects of the remaining governing parameters are to be considered in the following sub-sections.

Table 1 Comparison of the obtained results with those presented in (Goyeau et al. 1996; Bennacer et al. 2001; Bourich et al. 2004; Liu et al. 2008) in terms of Nusselt and Sherwood numbers for $\mathrm{N}=0$ and $\mathrm{Le}=10$.

\begin{tabular}{lcccc}
\hline & $\mathrm{R}_{\mathrm{T}}$ & 100 & 200 & 400 \\
\hline Goyeau et al. & $N u$ & 3.11 & 4.96 & 7.77 \\
1996 & $S h$ & 13.25 & 19.86 & 28.41 \\
Bennacer el al. & $N u$ & 3.11 & 4.96 & 7.77 \\
2001 & $S h$ & 13.24 & 19.83 & 29.36 \\
Bourich et al. & $N u$ & 3.11 & 4.96 & --- \\
2004 & $S h$ & 13.27 & 20.02 & --- \\
\hline \multirow{2}{*}{ Liu et al. 2008 } & $N u$ & 3.10 & 4.96 & 7.71 \\
& $S h$ & 13.23 & 19.93 & 29.76 \\
\hline \multirow{2}{*}{ Present work } & $N u$ & 3.12 & 5.01 & 7.87 \\
& $S h$ & 13.28 & 19.76 & 28.71 \\
\hline
\end{tabular}

Table 2 Validation of the numerical code in the case of a porous enclosure with localized heating and salting (Liu et al. 2008) for $\mathrm{Ra}_{\mathrm{T}}=100, \mathrm{Le}=10$ and different

\begin{tabular}{llccc}
\multicolumn{5}{c}{ values of $\mathrm{N}}$. \\
\hline & $\mathrm{N}$ & -20 & 0 & 20 \\
\hline \multirow{2}{*}{ Liu et al. 2008 } & $\Psi_{\max }$ & 0 & 2.836 & 2.777 \\
& $\Psi_{\min }$ & -0.477 & 0 & 0 \\
\hline \multirow{2}{*}{ Present work } & $\Psi_{\max }$ & 0 & 2.852 & 2.801 \\
& $\Psi_{\min }$ & -0.495 & 0 & 0 \\
\hline
\end{tabular}

Table 3 Grid sensitivity analysis for $\mathrm{N}=0, \mathrm{Le}=10$ and various values of $\mathrm{Ra}$

\begin{tabular}{ccccc}
\hline grid & $\mathrm{Ra}$ & $\mathrm{Nu}$ & $\mathrm{Sh}$ & $\Psi_{\max }$ \\
\hline \multirow{3}{*}{$61 \times 61$} & 100 & 3.127 & 13.182 & 4.704 \\
& 200 & 5.018 & 19.602 & 7.531 \\
& 400 & 7.822 & 28.572 & 11.728 \\
\hline \multirow{3}{*}{$81 \times 81$} & 100 & 3.123 & 13.280 & 4.706 \\
& 200 & 5.016 & 19.764 & 7.538 \\
& 400 & 7.877 & 28.710 & 11.753 \\
\hline \multirow{3}{*}{$101 \times 101$} & 100 & 3.120 & 13.372 & 4.707 \\
& 200 & 5.009 & 20.203 & 7.541 \\
& 400 & 7.891 & 28.896 & 11.906 \\
\hline
\end{tabular}




\subsection{Steady-state Solutions}

Typical steady-state solutions $(a=0)$ are illustrated for Le and $\mathrm{N}$ varying respectively in the ranges $0.1 \leq \mathrm{Le} \leq$ 10 and $-5 \leq \mathrm{N} \leq+5$. Hence, streamlines, isotherms and iso-concentrations, corresponding to different steadystate solutions, are presented in Figs. 2a-2e for Le $=10$, $\mathrm{R}_{\mathrm{T}}=400$ and various values of $\mathrm{N}$. By varying the buoyancy ratio, fundamental changes in the flow structure and temperature and concentration distributions are observed. Hence, by decreasing $\mathrm{N}$ from 4 (Fig. 2a) to -4 (Fig. 2e), the flow passes from unicellular clockwise flow to unicellular trigonometric one through different states characterized by a competition between counter-rotating cells. In fact, Fig. 2a exemplifies a typical feature of aiding double diffusive flow $(\mathrm{N}=+4)$. For this case, the flow near the heating and salting wall is driven vertically upward, and meanwhile, the low temperature and concentration at the right hand wall cause the fluid near it to sink. Both thermal and solutal buoyancy effects are added to each other and lead to an acceleration of the clockwise monocellular flow. The figure shows also important horizontal gradients of concentration near the vertical walls testifying of the presence of distinct solutal boundary layers. The corresponding isotherms are those of a convective dominating regime with relatively important thermal gradients at the level of the lower/upper part of the left/right vertical wall, i.e. in the region where the cooled/heated flow enters in contact with hot/cold wall. The decrease of $\mathrm{N}$ inside its positive range is followed by a decrease of the intensity of the monocellular clockwise flow. However, when $\mathrm{N}$ is decreased progressively starting from 0 , the effects of thermal and solutal buoyancy forces become opposite, which indicates that a decrease of $\mathrm{N}$ below zero is not favorable to the clockwise circulation. Thus, for $\mathrm{N}=$ -1 , a counterclockwise diagonal cell, joining the lower right and the upper left corners of the cavity is observed in Fig. $2 b$, forcing the breakdown of the large clockwise cell into two cells located at two opposite corners of the cavity. The isotherms show weak temperature gradients in the vicinity of the vertical boundaries indicating a dominating conductive regime. The corresponding isoconcentrations show that the mass transfer between the vertical walls and the fluid occurs mainly through the diagonal cell. A further decrease of $\mathrm{N}$ is largely favorable to the negative counterclockwise cell and this, to the detriment of the positive ones which are forced to occupy reduced spaces in the vicinity of the horizontal walls as it can be seen in Figs. 2c-2d, obtained respectively for $\mathrm{N}=-1.8$ and -1.95 . For $\mathrm{N}=-4$, the positive cells disappear completely and the flow pattern consists of a negative counterclockwise rotating cell (Fig. 2e). The concentration gradients are important in the upper and lower parts of the vertical left and right walls, respectively. The counterclockwise rotating cell is sliding the heated wall. It should be noted that the streamlines, the isotherms and the iso-concentrations exhibit a perfect symmetry with respect to the center of the cavity (centro-symmetry).

Variations of the overall Nusselt and Sherwood numbers versus $\mathrm{N}$ are presented respectively in Figs. $3 a-3 b$ for $R_{T}=400$ and various values of Le. The graphs clearly indicate that, for aiding flows $(\mathrm{N}>0)$, the transport rates of both heat and mass increase with the magnitude of $\mathrm{N}$, for all considered values of the Lewis number. Below zero (opposing flows), the transport rates of heat and mass are observed to decrease with the magnitude of $|\mathrm{N}|$. They are lower when compared with those generated in the case of aiding buoyancy forces for the same numerical values of $|\mathrm{N}|$. In addition, Sherwood and Nusselt number present minimum values $\left(\mathrm{Nu}_{\min }=1\right.$ independently of Le but $\mathrm{Sh}_{\min } \geq 1$ dependently of Le) when the two buoyancy forces are equal and opposing each other $(\mathrm{N}$ $=-1)$. Also, a perfect symmetry with respect to the vertical line $\mathrm{N}=-1$ is observed in the case of Sh while this symmetry is absent in the case of $\mathrm{Nu}$. By decreasing $\mathrm{N}$ (i.e. $\mathrm{N}$ increases in absolute values), the magnitude of the solutal buoyancy force increases and overpowers the upward thermal buoyancy one. Correspondingly, the transport rates begin to increase because it is the magnitude of the velocity but not its direction that influences the heat and mass transfer rates. The average Nusselt and Sherwood numbers corresponding to opposing buoyancy forces $(\mathrm{N}<0)$ are generally lower than those obtained with aiding buoyancy forces $(\mathrm{N}>0)$ since lower fluid velocities are generated in the former case. Finally, it is to be outlined that, for a given value of $\mathrm{N}$, any increase of the Lewis number (increase of mass diffusivity with respect to thermal diffusivity) is accompanied by an increase/decrease of mass/heat transfer.

\subsection{Unsteady Solutions}

In the unsteady regime, the attention is mainly focused on the effect of the amplitude, $a,(0 \leq a \leq 1)$ and the period, $\tau,(0.0001 \leq \tau \leq 10)$ of the variable temperature while the normalized porosity of the porous medium, the Lewis number and the Rayleigh-Darcy number were maintained constant at $0.8,10$ and 400, respectively. In the following, the results obtained are presented in terms of temporal variations of Nusselt $(\mathrm{Nu}(\mathrm{t}))$ and Sherwood $(\mathrm{Sh}(\mathrm{t}))$ numbers and maximum stream function $\left(\Psi_{\max }(t)\right)$. The mean values of these quantities, averaged in time over flow cycles, are also presented to point out the impact of the variable temperature on heat and mass transfers.

In the case of aiding buoyancy forces $(\mathrm{N}=4)$, the effect of the period $\tau$ is illustrated in Figs. $4 \mathrm{a}-5 \mathrm{c}$, where the evolutions versus time of $\mathrm{Nu}, \mathrm{Sh}$ and $\Psi_{\max }$ are presented for $a=1, \mathrm{Le}=10$ and $\mathrm{R}_{\mathrm{T}}=400$. Globally, the results show that the system reply to the external periodic excitation is periodic with periods identical to those of the imposed variable temperature. In addition, by increasing $\tau$, the sinusoidal nature of the excitation is preserved in the evolutions of $\mathrm{Nu}(\mathrm{t})$ while it is visibly lost in the case of $\operatorname{Sh}(\mathrm{t})$ and $\Psi_{\max }(\mathrm{t})$. Also, it is important to note that the mean values of heat transfer peak for one of the three selected periods $(\overline{\mathrm{Nu}}(\tau=$ $0.01)=14.75, \overline{\mathrm{Nu}}(\tau=0.05)=15.26$ and $\overline{\mathrm{Nu}}(\tau=0.1)$ $=14.92)$. This behavior indicates that the resonance phenomenon is possible and corresponds to a critical value of $\tau$ (to be determined). The trends observed remain qualitatively similar for lower values of the 
amplitude $a$; the increase of the latter is accompanied by an amplification of the results obtained. It is to note from Fig. 4a that the mean instantaneous Nusselt number becomes negative during some range of time in each flow cycle. This can be explained by the fact that, during the heat transfer process, the temperature of the exciting wall drops and becomes lower than that of the fluid in its vicinity. This means that, a part of the heat gained by the fluid in early stages is evacuated by the system through its hot wall when its temperature drops.

Based on a set of preliminary tests, time averaged values of the Nusselt number and the maximum stream function $\left(\overline{\mathrm{Nu}}\right.$ and $\left.\bar{\Psi}_{\max }\right)$, obtained for various periods, let suppose the existence of the resonance phenomenon within the porous medium. This phenomenon, reported in the past by several authors in enclosures filled with fluids (Lage and Bejan 1993; Lakhal et al. 1999; EL Ayachi et al. 2008) or with saturated porous media (Antohe and Lage 1996; Antohe and Lage 1997) in the absence of mass transfer, is characterized by maximum fluctuations in the response of the system to an external excitation with well defined period. Hence, variations of $(\overline{\mathrm{Nu}}, \overline{\mathrm{Sh}})$ and $\left(\bar{\Psi}_{\text {max }}\right.$, $\bar{\Psi}_{\text {min }}$ ) with the period $\tau$ of the variable temperature are presented respectively in Figs. $5-6$ for $\mathrm{N}=+4$ and various values of the amplitude $a$. Results corresponding to the case of the isothermal heating source $(a=0)$ are also presented in the same figures as references. It is to be specified that imposed thermal excitations with periods lower than $\tau \leq 0.0003$ have no detrimental effect on mean heat and mass transfers (Figs. 5a-5b) since their values remain practically identical to those obtained in the absence of thermal modulation. Thereafter, $\overline{\mathrm{Nu}}$ increases with $\tau$ until reaching a maximum value for a critical value of $\tau \approx$ 0.05 which is not affected by the increase of the oscillating amplitude $a$. Above this threshold, a further increase of $\tau$ leads to a slight decrease of the Nusselt number towards almost asymptotic values which are dependent on the amplitude of the imposed thermal excitation. By increasing the parameter $a$, the resonance phenomenon in the case of heat transfer becomes more pronounced when this parameter is larger than 0.25 and the fluctuating amplitude increases monotonically. Variations of the Sherwood number show that this quantity reaches a minimum for a critical period of 0.02 . The variation of $\bar{\Psi}_{\max }$ with $\tau$, presented in Fig. $6 \mathrm{a}$, shows monotonous increase towards maximum reached around $\tau=0.05$. Beyond this threshold value, a decrease of $\bar{\Psi}_{\max }$ is observed until reaching asymptotic values, lower than that obtained for the case of a constant heating temperature. In addition, it can be seen that the increase of the amplitude $a$ leads to an intensification of the fluid. This tendency is reversed for $\tau \geq 0.7$. For lower values of the period $\tau$ and $a \geq 0.75$, it can be seen from Fig. $6 \mathrm{~b}$ that weak negative values of the stream function $\left(\Psi_{\min } \neq 0\right)$ were obtained when the heating temperature is periodically varied. Hence, during the evolution of the flow cycle, the flow structure recovers states where the fluid flow consists of the presence of a dominating clockwise rotating flow and a weak secondary trigonometric flow (playing a negligible role) which reaches its maximum intensity for $\tau=0.001$ and disappears completely for values of $\tau$ higher than 0.05 for $a=1$.

In the case of opposing buoyancy forces $(\mathrm{N}=-4)$, variations of averaged Nusselt and Sherwood numbers, presented in Figs. 7a-7b, show that the increase of the amplitude and the period of the exciting temperature acts in the sense to affect negatively heat and mass transfer across the cavity in comparison with the steady state solution $(a=0)$. The resonance phenomenon is seen to be absent for opposing buoyancy forces. It can be also reported that these functions remain close to their respective steady values in the case of weak periods $(\tau \leq 0.0002)$ indicating an insensitivity of the system vis-à-vis to the imposed thermal modulation. Variations of the averaged values of the maximum ( $\left.\bar{\Psi}_{\max }\right)$ and minimum $\left(\bar{\Psi}_{\min }\right)$ stream functions, presented respectively in Figs. $8 \mathrm{a}-8 \mathrm{~b}$, show monotonous increase/decrease of $\bar{\Psi}_{\max } / \bar{\Psi}_{\text {min }}$ towards maximum/minimum. Beyond this threshold, a decrease/increase of $\bar{\Psi}_{\max } / \bar{\Psi}_{\text {min }}$ is observed towards values identical/lower than those of the steady state solution in the case of $\bar{\Psi}_{\max } / \bar{\Psi}_{\min }$. It is to underline that, for each value of $\tau$, the fluid flow is characterized by a dominating counterclockwise cell. Also, the fluid circulation is seen to be more enhanced with the increase of the amplitude of the variable temperature.

\section{Conclusion}

Two-dimensional transient double diffusive natural convection in a square porous enclosure saturated with a binary fluid, heated from one vertical wall by imposing variable thermal boundary conditions and submitted to imposed constant concentrations, is studied numerically. Results obtained show that the flow structure is strongly affected by the buoyancy ratio forces N. More precisely, the clockwise and counterclockwise flow patterns are seen to be respectively favoured by larger positive and negative values of $\mathrm{N}$. The temperature and concentration fields are seriously affected by varying the parameter N. It is also observed that an increase of the Lewis number is accompanied by an increase of the mass transfer but it leads to a reduction of heat transfer across the cavity. By imposing a sinusoidal exciting temperature to the system, the heat transfer and fluid circulation within the cavity are enhanced noticeably in comparison with the standard case of non-oscillating temperature condition. The time-averaged heat transfer and flow intensity reach maxima for a critical value of the heating period and the latter is independent of the amplitude of the variable temperature. This resonance phenomenon is more amplified by increasing the amplitude of the exciting temperature in the case of aiding buoyancy forces $(\mathrm{N}>0)$. In the mean time, the resonance phenomenon is not observed in the case of mass transfer and the latter passes by a minimum value when the heat transfer resonates. In the case of opposing buoyancy forces $(\mathrm{N}<0)$, an increase of the amplitude and the period of the variable temperature affects 
negatively the heat and mass transfers across the cavity in comparison with constant heating temperature.

\section{REFERENCES}

Abourida, B., M. Hasnaoui and S. Douamna (1999). Transient natural convection in a square enclosure with horizontal walls submitted to periodic temperatures. Numerical Heat Transfer A 36, $737-$ 750.

Amahmid, A., M. Hasnaoui, M. Mamou, P. Vasseur (1999a). Boundary layer flows in a vertical porous enclosure induced by opposing buoyancy forces. Int. J. Heat Mass Transfer 42, 3599-3608.

Amahmid, A., M. Hasnaoui, M. Mamou, P. Vasseur (1999b). Double-diffusive parallel flow induced in a horizontal Brinkman porous layer subjected to constant heat and mass fluxes. Int. J. Heat Mass Transfer 35, 409-421.

Antohe, B.V. and J.L. Lage (1996). Amplitude effect on convection induced by time-periodic horizontal heating. Int. J. Heat Mass Transfer 39, 1121-1133.

Antohe, B.V. and J.L. Lage (1997). The Prandtl number effect on the optimum heating frequency of an enclosure filled with fluid or with a saturated porous medium. Int. J. Heat Mass Transfer 40, 1313-1323.

Bennacer, V., A.A. Mohamad and D. Akrour (2001). Transient natural convection in an enclosure with horizontal temperature and vertical solutal gradients. Int. J. Thermal Sci. 40, 899-910.

Bourich, M., M. Hasnaoui and A. Amahmid (2004). Double-diffusive natural convection in a porous enclosure partially heated from below and differentially salted. Int. J. Heat Fluid Flow 25, 1034-1046.

Douamna, S., M. Hasnaoui and B. Abourida (2000). Two-dimensional transient natural convection in a repetitive geometry submitted to variable heating from below: numerical identification of routes leading to chaos. Numerical Heat Transfer A 37, 779-799.

El Ayachi, R., A. Raji, M. Hasnaoui and A. Bahlaoui (2008). Combined effect of radiation and natural convection in a square cavity differentially heated with a periodic temperature. Numerical Heat Transfer A 53, 1339-1356.

Goyeau, B., J.P. Songbe and D. Gobin (1996). Numerical study of double-diffusive natural convection in a porous cavity using the DarcyBrinkman formulation. Int. J. Heat Mass Transfer $39,1363-1378$

Ingham, D.B. and I. Pop (2005). Transport Phenomena in Porous Media. Oxford, Elsevier.
Lage, J.L. and A. Bejan (1993). The resonance of natural convection in a horizontal enclosure heated periodically from the side. Int. J. Heat Mass Transfer 36, 2027-2038.

Lakhal, E.K., M. Hasnaoui and P. Vasseur (1999). Numerical study of transient natural convection in a cavity heated periodically with different types of excitations. Int. J. Heat Mass Transfer 42, 39273941.

Liu, D., F.Y. Zhao and G.F. Tang (2008). Thermosolutal convection in saturated porous enclosure with concentrated energy and solute sources. Energy Conversion and Management 49, 16-31.

Malashetty, M.S. and V.S. Wadi (1999). RayleighBenard convection subject to time dependent wall temperature in a fluid saturated porous layer. Fluid Dyn. Res. 24, 293-308.

Malashetty, M.S. and D. Basavaraja (2002). RayleighBenard convection subject to time dependent wall temperature/gravity in a fluid-saturated anisotropic porous medium. Heat Mass Transfer 38, 551-563.

Mamou, M., P. Vasseur and E. Bilgen (1995). Multiple solutions for double-diffusive convection on a vertical porous enclosure. Int. J. Heat Mass Transfer 38, 1787-1798.

Nield, D.A. and A. Bejan (1999). Convection in Porous Media. New York. Springer-Verlag.

Nishimura, M., M. Wakamatsu and A.M. Morega (1998). Oscillatory double-diffusive convection in a rectangular enclosure with combined horizontal temperature and concentration gradients. Int. J. Heat Mass Transfer 41, 1601-1611.

Sezai, I. and A.A. Mohamad (1999). Three-dimensional double-diffusive convection in a porous cubic enclosure due to opposing gradients of temperature and concentration. J. Fluid Mech. 400, 333-353.

Trevisan, O.V. and A. Bejan (1987). Mass and heat transfer by high Rayleigh number convection in a porous medium heated from below. Int. J. Heat Mass Transfer 30, 2341-2356. 

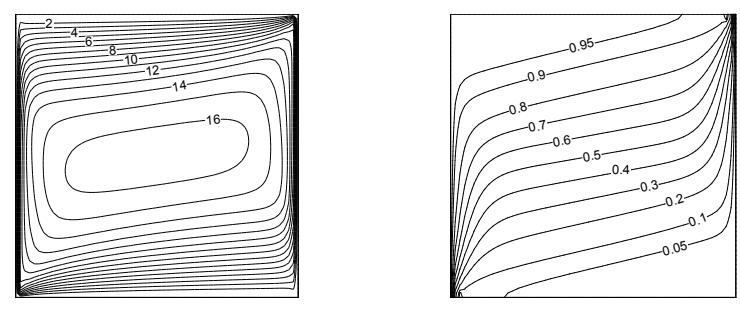

(a) $\mathrm{N}=+4$
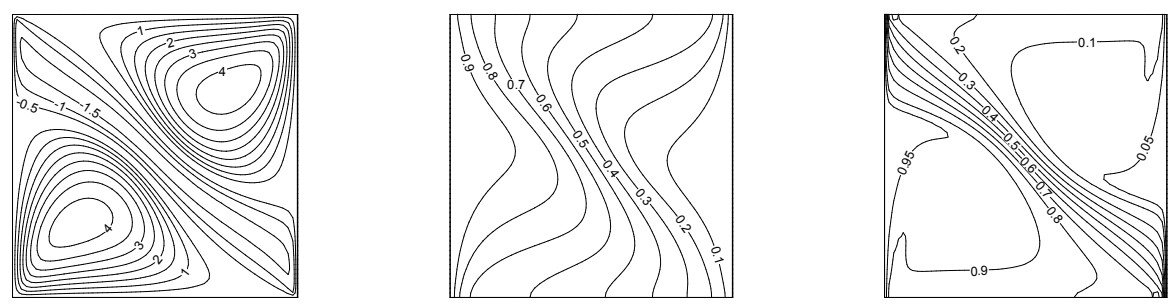

(b) $\mathrm{N}=-1$
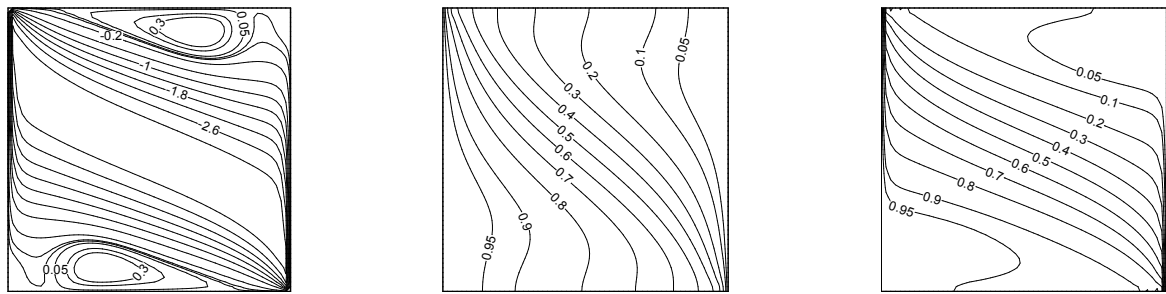

(c) $\mathrm{N}=-\mathbf{1 . 8}$
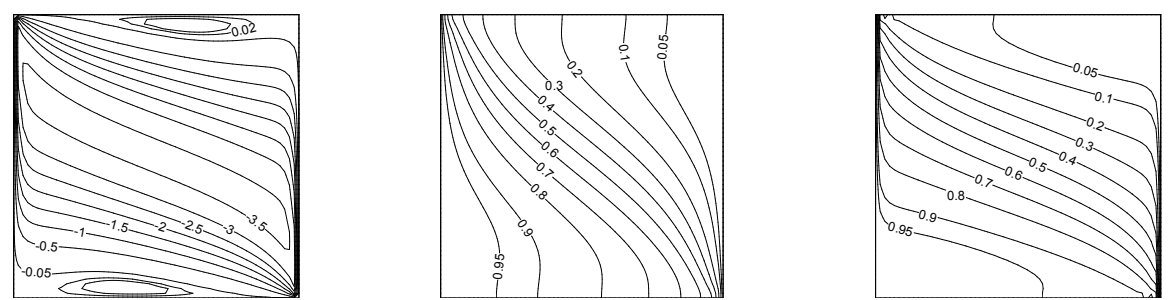

(d) $\mathrm{N}=-\mathbf{1 . 9 5}$
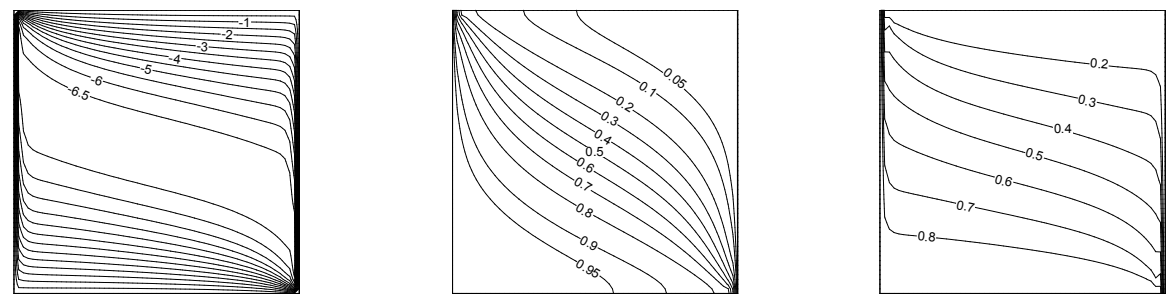

(e) $\mathrm{N}=-4$

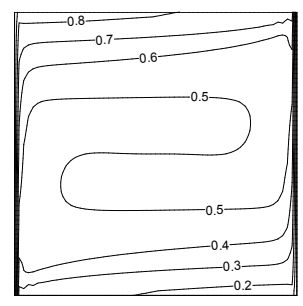




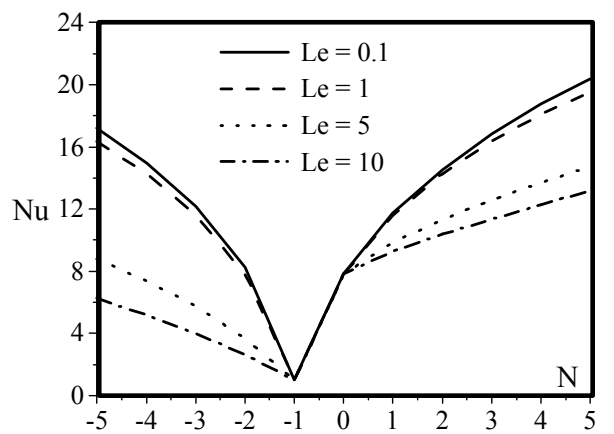

(a)

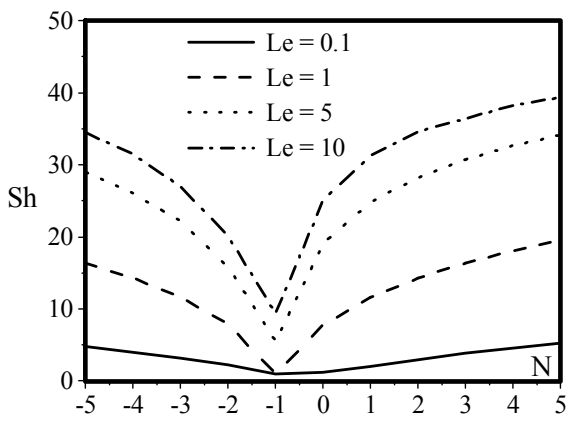

(b)

Fig. 3. Overall Nusselt number $N u$ (a) and Sherwood number $S h$ (b) as functions of buoyancy ratio $\mathrm{N}$ for the case $\mathrm{R}_{\mathrm{T}}=400$ with the Lewis number as parameter.
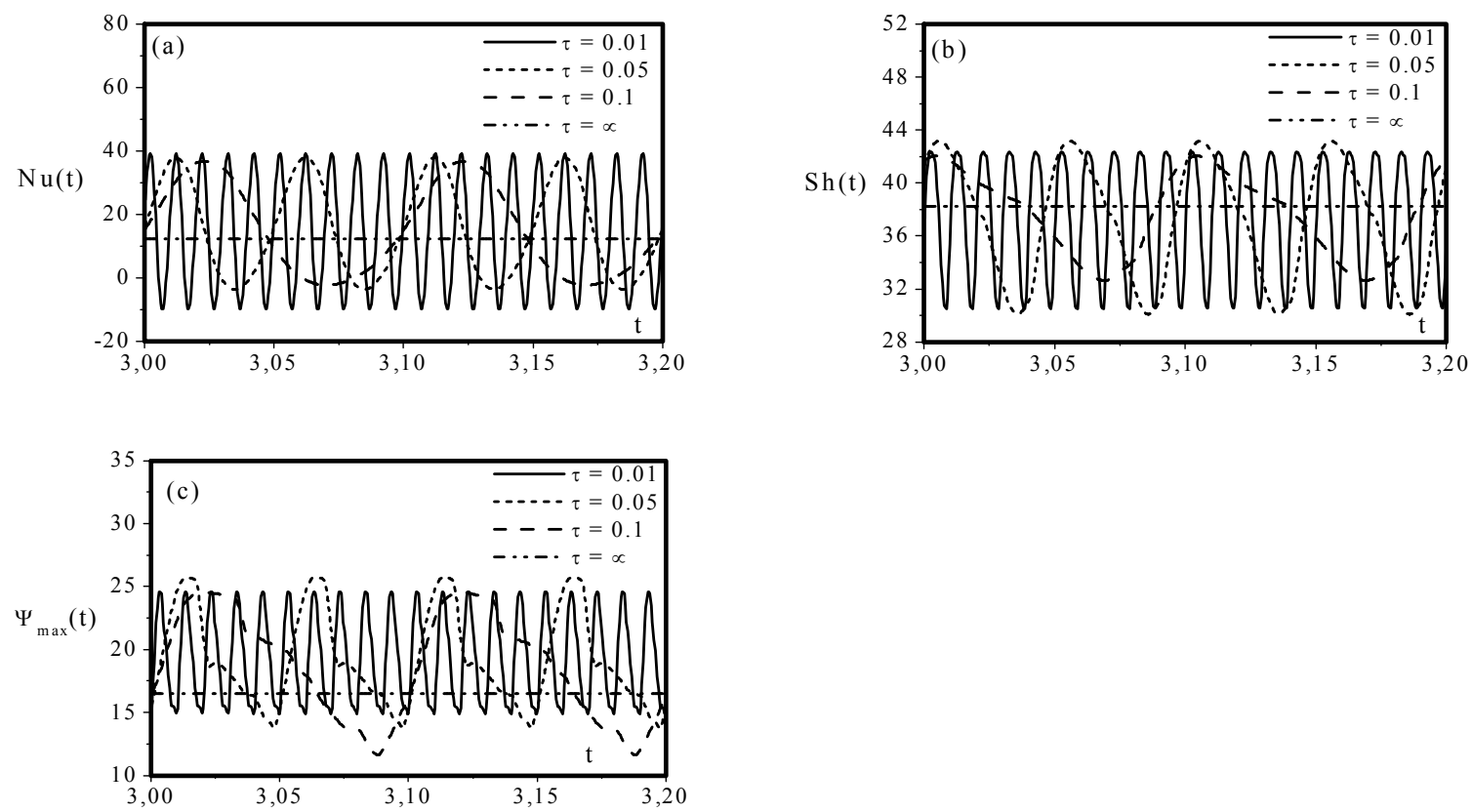

Fig. 4. Effect of the period $\tau$ on the time history of Nusselt number (a), Sherwood number (b) and maximum stream function (c) for $a=1, \mathrm{Le}=10, \mathrm{~N}=+4$ and $\mathrm{R}_{\mathrm{T}}=400$.

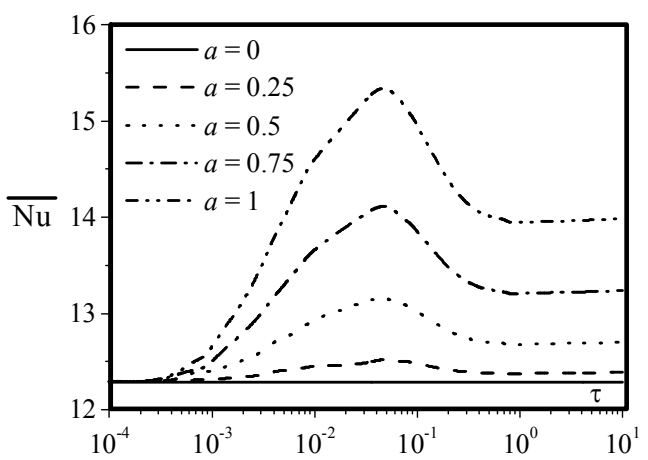

(a)

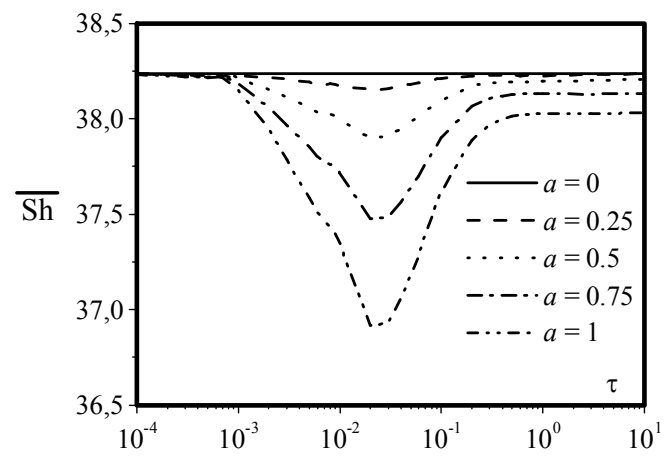

(b)

Fig. 5. Variation of the average Nusselt number (a) and Sherwood number (b) with the period $\tau$ for Le $=$ $10, \mathrm{~N}=+4, \mathrm{R}_{\mathrm{T}}=400$ and different values of the amplitude $a$. 


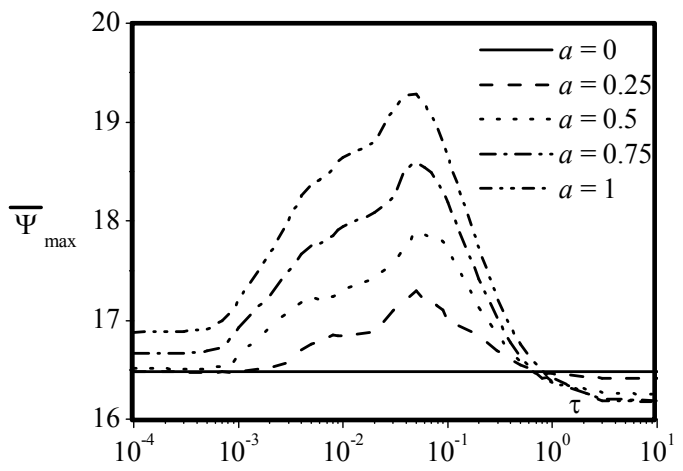

(a)

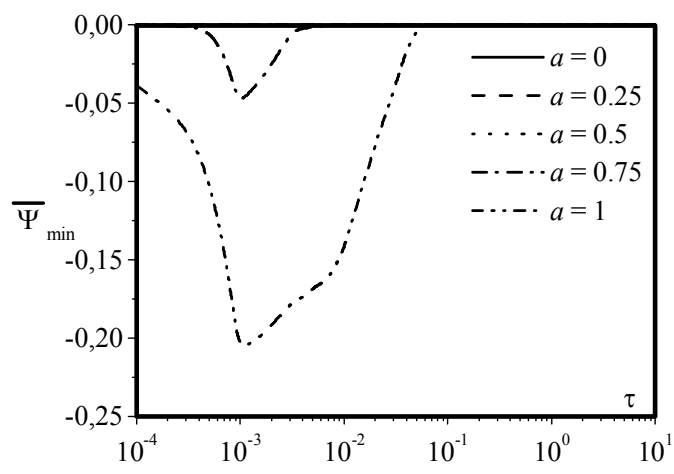

(b)

Fig. 6. Variation of the maximum (a) and minimum (b) stream functions with the period $\tau$ for $\operatorname{Le}=10$, $\mathrm{N}=+4, \mathrm{R}_{\mathrm{T}}=400$ and different values of the amplitude $a$.

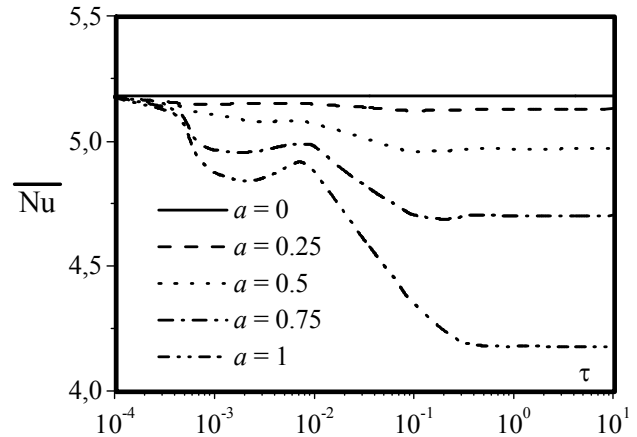

(a)

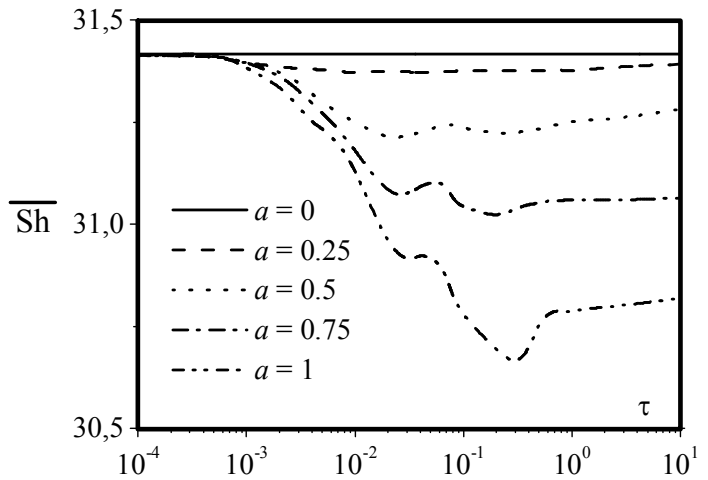

(b)

Fig. 7. Variation of the average Nusselt number (a) and Sherwood number (b) with the period $\tau$ for $\mathrm{Le}=10, \mathrm{~N}=-4, \mathrm{R}_{\mathrm{T}}=400$ and different values of the amplitude $a$.

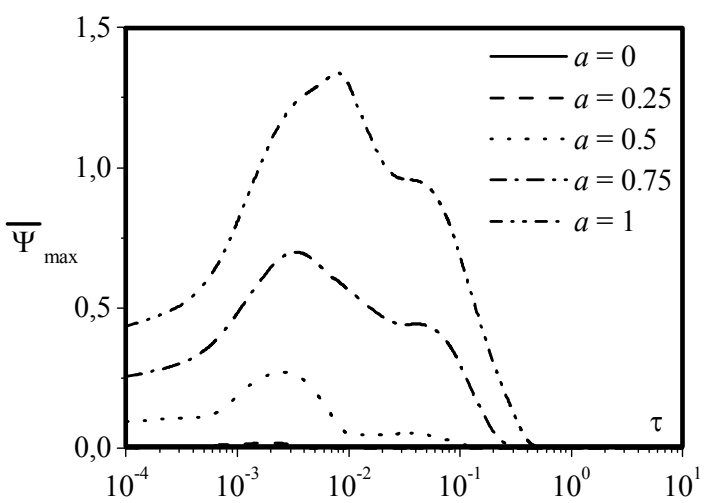

(a)

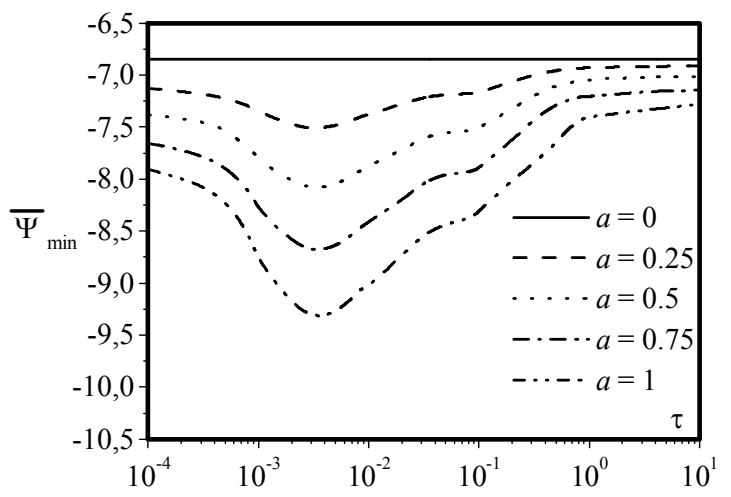

(b)

Fig. 8. Variation of the maximum (a) and minimum (b) stream functions with the period $\tau$ for $\mathrm{Le}=10$, $\mathrm{N}=-4, \mathrm{R}_{\mathrm{T}}=400$ and different values of the amplitude $a$. 